\title{
VARIABILIDADE HORIZONTAL DE ATRIBUTOS DE FERTILIDADE E AMOSTRAGEM DO SOLO NO SISTEMA PLANTIO DIRETO(1)
}

\author{
J. A. SCHLINDWEIN ${ }^{(2)} \&$ I. ANGHINONI(3)
}

\begin{abstract}
RESUMO
As adubações, a lanço, quando desuniformes, ou as em linhas, no sistema plantio direto, aumentam a variabilidade dos atri butos quími cos do solo. Nestas condições, há necessidade de se saber qual a mel hor forma e o número de subamostras por coletar para uma boa representatividade da fertilidade da área a ser cultivada. $O$ objetivo deste estudo foi quantificar a variabilidade horizontal de atributos de ferti lidade do solo no sistema plantio di reto com diferentes modos de adubação e tempos de cultivo, com vistas em definir o número de subamostras necessárias para formar uma amostra representativa da fertilidade do solo de uma área. Foram coletadas, em novembro de 1997, 36 amostras si mples, com pá de corte, na camada de $0-10 \mathrm{~cm}$, de forma diferenciada nas adubações a lanço ( 5 e $10 \mathrm{~cm}$, espessura da fatia e largura) e em linhas ( $5 \mathrm{~cm}$ de espessura da fatia pela largura das entrelinhas de semeadura da últi ma cultura), em oito lavouras comerciais na região noroeste do Rio Grande do Sul. 0 número mínimo de subamostras para esti mar com boa representatividade $(\alpha=0,05$ e erro em relação à média de 10\%) o pH, o índice SMP e o teor de matéria orgânica foi baixo (menor do que 8) e alto (maior do que 40) para fósforo e potássio (Mehlich-1). 0 número de subamostras indicado para o sistema convencional (20) pode ser usado no sistema plantio direto, desde que seja admitido um erro de $20 \%$ em relação à média.
\end{abstract}

Termos de indexação: atributos químicos, número de subamostras, formas de adubação.

\footnotetext{
(1) Parte da Tese de Mestrado apresentada pelo primeiro autor à Universidade F ederal do Rio Grande do Sul - UF RGS para obtenção do título de Mestre em Ciência do Solo. Financiado pela FAPERGS e FINEP/PRONEX. Recebido para publicação em maio de 1999 e aprovado em novembro 1999.

(2) Engenheiro-Agrônomo MsC. UniversidadeFederal do Rio Grande do Sul - UFRGS. Caixa Postal 776, CEP $90001-970$ Porto Alegre (RS).E-mail: jairojas@vortex.ufrgs.br.

(3) Professor Adjunto da Faculdade de Agronomia da UFRGS. Bolsista do CNPq.
} 


\title{
SUMMARY: HORIZONTAL VARIABILITY OF SOIL FERTILITY ATTRIBUTES AND SOIL SAMPLING UNDER A NO-TILLAGE SYSTEM
}

\begin{abstract}
Non-uniformly broadcast as well as row applied fertilizers under a no-tillage system increase the variability of soil chemical attributes. Therefore, to acomplish an appropriate fertility description of an area under no-tillage it is necessary to establish better sampling procedures, including number of subsamples. So, the main objective of this work was to measurethehorizontal variability of soil fertility attributes, allowing, as a consequence, a definition of thenumber of subsamples needed to composea whol eand representativefertility sample. In November 1997, in eight chosen areas of commercial agriculture production in thestate of Rio GrandedoSul, Brazil, 36 singl esamples werecollected in 0-10 cm layer depths with a cutting shovel, with the slices being $5 \mathrm{~cm}$-thick, and $10 \mathrm{~cm}$-wide in broadcast areas and $5 \mathrm{~cm}$-thick/ interrow-widein row fertilized areas. Themini mum number of subsamples to acceptably estimate ( $\alpha=0,05$ and $10 \%$ mean error) $\mathrm{pH}$, SMP value and organic matter was low (less than 8) and high (morethan 40) for phosphorus and potassium (Mehlich-1). Thenumber of subsamples defined for conventional tillage(20) may also beused for thenotillage system, provided a $20 \%$ mean error is allowed.
\end{abstract}

Index terms: chemical characteristics, number of subsamples, fertilizati on methods.

\section{INTRODUÇÃO}

A utilização do solo no sistema convencional de cultivo altera seus atributos químicos originais, pela aplicação de corretivos e fertilizantes (Santos \& Vasconcellos, 1987; Couto 1997). Nosistema plantio direto, a variabilidade do solo é aumentada, ainda mais, pela ação residual das linhas de adubação, que se mantêm na seqüência dos cultivos, juntamente com a redistribuição dos nutrientes reciclados dos resíduos (Souza, 1992; Couto, 1997; Anghinoni \& Salet, 1998).

O aumento da área cultivada no sistema plantio direto no Brasil está ocorrendo em maiores proporções na década de 90 , em relaçãoàs anteriores, atingindo, em 1996, 5,2 milhões de ha, aproximadamente $10 \%$ da área total cultivada (Wiethölter et al., 1998). No estado do Rio Grande do Sul, esse incremento foi mais pronunciado, atingindo, em 1997, 2,3 milhões de ha, o que representa 40\% da área total cultivada (Farias \& Ferreira, 1997). Existem regiões nesse estado, onde o incremento é ainda maior, como nas do Planalto Médio e Alto Uruguai, com mais de $90 \%$ de suas áreas cultivadas nesse sistema (Denardin et al., 1998).

A distribuição superficial uniforme de cal cário e da palhada das culturas no sistema plantio direto leva à baixa variabilidade horizontal nos atributos de acidez do sol o e nos teores de matéria orgânica, o que determina um pequeno número de subamostras para a coleta de amostras de solo representativas da área (Souza, 1992; Salet et al., 1996; Couto, 1997; Schlindwein et al., 1998). Entretanto, as adubações a lanço, quando desuniformes, ou em linhas, favorecem a variabilidade horizontal de nutrientes, como fósforo e potássio, que ficam disponíveis por mais de uma cultura (J ames \& Dow, 1972; K lepker \& Anghinoni, 1993, 1995; Kray et al., 1997). Nestecaso, o número de subamostras necessárias para a coleta de amostras representativas da lavoura torna-se maior.

Segundo Schlindwein et al. (1998), o instrumento utilizado na amostragem do solo também pode ser fonte de variabilidade, considerando o próprio tamanho (volume de solo) da subamostra, como também possíveis perdas, como, por exemplo, na col eta com trado de rosca, que pode acarretar perdas de solo, principalmente das camadas superficiais, mais ricas em al guns dos atributos quími cos do solo.

A rotação de culturas éuma condição básica para o sucesso e continuidade do sistema plantio direto. Culturas, com diferentes espaçamentos, são cultivadas numa mesma área, e as linhas de adubação quase sempre não coincidem. Esta prática sugere, segundo Anghinoni \& Salet (1998), que o sistema plantio direto consolidado (mais do que 5 anos) apresenta variabilidade menor do quena fase inicial de implantação e, provavelmente, mais próxima da variabilidade do sistema convencional de preparo do solo.

Considerando esses fatores potenciais de variabilidade no sistema plantio direto, torna-se necessário definir métodos de amostragem representativos quanto ao sítio de col eta, quanto ao tamanho (volume) e quanto ao número de subamostras necessárias para contemplar tal variabilidade dentro de critérios (parâmetros) de confiabilidadeestatística.

O objetivo deste trabalho foi determinar a magnitude da variabilidade horizontal dos índices 
de fertilidade dos solos utilizados no Programa de Adubação e Calagem dos estados do Rio Grande do Sul e Santa Catarina (Comissão..., 1995), em lavouras comerciais, considerando diferentes solos, modos de adubação etempos decultivo, para definir o número de subamostras de solo por coletar em lavouras no sistema plantio direto, dentro de determinados limites de precisão.

\section{MATERIAL E MÉTODOS.}

Amostras de sol os foram retiradas durante o mês de novembro de 1997 em oito lavouras localizadas na região Noroeste do Rio Grande do Sul, nos municípios de Santo Augusto, Coronel Bicaco, São Valério do Sul e Campo N ovo. Os solos, originários dederramamentos basálticos, são de textura argilosa e classificados como Latossolo Vermelho distrófico típico, Latossolo Vermelho distroférrico típico e Chernossolo Argilúvico férrico típico (EMBRAPA, 1999).

Nas lavouras selecionadas, foram demarcadas áreas, que variaram de dois a quatro ha, consideradas homogêneas quanto ao tipo de solo, cor, textura, topografia e histórico de cultivo. As amostras foram retiradas em 36 pontos (amostras simples), distribuídos al eatoriamente nessas áreas. Utilizouse pá de corte para a coleta de amostras, com $5 \mathrm{~cm}$ de espessura da fatia e $10 \mathrm{~cm}$ de largura, nas lavouras com adubação a lanço, e $5 \mathrm{~cm}$ de espessura da fatia pela largura das entrelinhas do ultimo cultivo (soja: $45 \mathrm{~cm}$ e milho: $80 \mathrm{~cm}$ ), nas lavouras com adubação em linhas. A amostragem foi feita na camada de 0-10 $\mathrm{cm}$ de profundidade.

As amostras dos solos, após secas ao ar e peneiradas, foram analisadas de acordo com os procedimentos descritos por Tedesco et al. (1995). Foram determinados o pH em água, índice SMP, utilizado para recomendação da necessidade de calcário pela Comissão... (1995), teor deargila, fósforo e potássio disponíveis (Mehlich-1) e matéria orgânica, por digestão úmida e determinação colorimétrica. As anál ises foram feitas em duplicata nolaboratório, tendo sido utilizados os dados médios na apresentação dos resultados.

Na determinação do número mínimo de subamostras, para formar uma amostra composta e representativa, foram utilizados os resultados analíticos das amostras simples para o cálculo da variabilidade dos índices de fertilidade do solo e dos parâmetros estatísticos indicados na Equação (1), utilizada por Petersen \& Calvin (1986), Santos \& Vasconcellos (1987), Souza (1992), Davis et al . (1995) e Couto (1997), com algumas diferenças quanto à sua denominação e definição.

$$
\mathrm{n}=\left[\left(\mathrm{t}_{\alpha} \cdot \mathrm{CV}\right) / \mathrm{e}\right]^{2}
$$

em que $\mathbf{n}$ é o número médio de subamostras; $\mathbf{C V}$ éo coeficiente de variação; t é o val or da tabela (testet) correspondente ao erro $\alpha$ e ao número de graus de liberdade do quadrado médio residual, e e é o erro em torno da média.

\section{RESULTADOS E DISCUSSÃO}

Os valores médios dos atributos de fertilidadedas amostras simples dos solos estão apresentados no quadro 1. Os teores de matéria orgânica estão, em sua maioria, na faixa "média" (25 a 50 g kg-1), considerando a classificação utilizada para o sistema de cultivo convencional nos estados do Rio Grande doSul eSanta Catarina (Comissão..., 1995). O menor valor $\left(25 \mathrm{~g} \mathrm{~kg}^{-1}\right)$ corresponde à lavoura com cinco anos de cultivo no sistema plantio direto, com teor de argila mais baixo e com histórico de menor manutenção da palhada no solo. A argila, que confere proteção física, físico-química e química para a matéria orgânica no solo (Sollins et al., 1996), pode ser um dos fatores responsáveis pelos diferentes teores encontrados. Além disso, os maiores teores de matéria orgânica foram encontrados nas lavouras com maior tempo de cultivo (10 e 12 anos) nosistema plantio direto indicam, como também observado por Bayer (1996), que os sistemas conservacionistas tendem a acumular matéria orgânica com o tempo de cultivo.

$\mathrm{O}$ pH do solo foi baixo em todas as lavouras, com os menores valores nos locais com maior tempo de cultivo (10 e 12 anos) sem aplicação do cal cário; o índice SMP, que mede as fontes potenciais de acidez do solo, foi variável entre as lavouras, indicando a necessi dade de 2,7 t ha-1 decal cário em três lavouras (Índice SMP 6,1) a 9,1 t ha-1 de calcário em uma lavoura (Índice SMP 5,1), para el evar opH do solo a 6,0 (Comissão..., 1995).

Os teores de potássio disponível (Mehlich-1) no sol o ficaram acima do nível crítico (80 mg kg-1) para a maioria das culturas, indicado para o sistema convencional de cultivo (Comissão..., 1995), bem como os teores de fósforo disponível (Mehlich-1), considerando o teor de argila adotado pela Comissão... (1995). N este caso, a não-mobilização do solo nas lavouras cultivadas no sistema plantio direto evita a exposição de novos sítios de adsorção das formas lábeis de fósforo (Sousa \& Volkweiss, 1987) e justificam a predominância dos teores el evados de fósforo disponível (Quadro 1). J á para o potássio, os valores el evados deveram-se, possivelmente, à menor lixiviação decorrente do acúmulo de matéria orgânica (maior CTC) e da remoção do potássi o de camadas subsuperficiais pel as raízes com liberação na superfície, pela lavagem das folhas (Klepker \& Anghinoni, 1995). A menor perda de solo por erosão e a adição de fósforo e potássio nas 
adubações, em quantidades maiores do que as exportações pelos grãos, também determinaram aumento desses nutrientes na camada superficial do solo com o tempo de cultivo no sistema plantio direto (Wiethölter et al., 1998).

A variabilidade dos valores de matéria orgânica, pH em água e índice SMP foi baixa (Quadro 1), com val ores médios dos coeficientes de variação menores do que $10 \%$ e com pequena amplitude entre as lavouras, especialmente para o índice SMP, que apresentou os valores mais baixos. No entanto, a variabilidade para o fósforo e potássio foi muito el evada, al cançando valores de coeficiente de variação de até $48 \%$ e grande amplitude de variação.

Os coeficientes de variação da matéria orgânica, fósforo e potássio disponíveis encontrados neste trabal ho atingiram a mesma magnitude dos obtidos por Souza (1992), em diferentes sistemas de cultivo, locais e profundidades de amostragem, e por Salet et al. (1996) eSchlindwein et al. (1998), em diferentes lavouras no sistema plantio direto. Foram, no entanto, inferiores aos obtidos nosistema convencional esuperi ores aos encontrados por Couto (1997) em solo com vegetação de cerrado, na camada de $0-20 \mathrm{~cm}$.

A baixa variabilidade horizontal dos índices de matéria orgânica, pH em água eSMP era esperada, uma vez que a distribuição da pal hada e do cal cário no solo é uniforme. Isto, entretanto, não ocorre na aplicação dos fertilizantes no sistema plantio direto, que é efetuada a cada cultivo, predominantemente em sulcos, nas linhas de semeadura, que se mantêm pouco al teradas com o tempo de cultivo (Kray et al.,
1997). Considerando que a variabilidade observada para os índices de fósforo e potássio neste trabalho não foi relacionada com o tempo de cultivo $\left(R^{2}\right.$ de $0,12^{\text {ns }}$ e 0,50 ns, respectivamente), a hipótese de maior uniformidade, pela não-coincidência das linhas de adubação, levantada por Anghinoni \& Salet (1998), não foi comprovada. Observou-se, somente, e a exemplo do relatado por Kray et al. (1997), uma tendência de diminuição da variabilidade com o aumento dos teores dos nutrientes, especialmente de potássio.

Os manuais dos diversos Programas de Adubação e Calagem, elaborados regionalmente no Brasil, pelas respectivas Comissões de F ertilidade do Solo (Anghinoni \& Volkweiss, 1984), recomendam coletar em torno de 20 subamostras para formar cada amostra composta e representativa de determinada área homogênea no sistema convencional de cultivo. Tais recomendações se referem a subamostras pequenas, normalmente coletadas com trado de rosca, calador (diâmetro de 2 a $3 \mathrm{~cm}$ ) ou a parte central da fatia retirada com pá de corte (em torno de 2,5 e 5,0 cm de espessura e de largura). Embora os limites de precisão nãotenham sido apresentados nesses manuais, acredita-se, com base nos diversos trabal hos sobre o assunto (Petersen \& Calvin, 1986; Santos \& Vasconcellos, 1987; Souza, 1992; Davis et al., 1995; Couto, 1997), que uma baixa probabilidade deerro ( $\alpha=0,05$ e erro eem relação à média de $10 \%)$ tenha si do adotada na E quação (1) para o cál culo do número (n) de subamostras. Assim, a coleta das 20 subamostras recomendada pel os manuais estará conferindograndeconfiabilidadeerepresentatividade

Quadro 1. Teores de argila, matéria orgânica e valores de pH, índice SMP e potássio e fósforo disponíveis em oito lavouras com diferentes formas de adubação e tempo de cultivo no sistema plantio direto, na região noroeste do RS

\begin{tabular}{|c|c|c|c|c|c|c|c|}
\hline \multicolumn{2}{|c|}{ Característica da lavoura } & \multirow{2}{*}{$\begin{array}{c}\text { Teor de } \\
\text { argila }\end{array}$} & \multicolumn{5}{|c|}{ Atributo de fertilidade do solo } \\
\hline $\begin{array}{l}\text { Forma de } \\
\text { adubação }\end{array}$ & $\begin{array}{l}\text { Tempo de } \\
\text { cultivo }\end{array}$ & & $\begin{array}{l}\text { Matéria } \\
\text { orgânica }\end{array}$ & $\begin{array}{l}\text { pH } \\
\text { água }\end{array}$ & $\begin{array}{l}\text { Índice } \\
\text { SMP }\end{array}$ & $\begin{array}{l}\text { Potássio } \\
\text { Mehlich-1 }\end{array}$ & $\begin{array}{l}\text { Fósforo } \\
\text { Mehlich-1 }\end{array}$ \\
\hline & anos & \multicolumn{2}{|c|}{$\mathrm{g} \mathrm{kg}^{-1}$} & & & \multicolumn{2}{|c|}{$\mathrm{mg} \mathrm{kg}^{-1}$} \\
\hline L anço & 6 & 500 & $40(12,6)^{(1)}$ & $5,6(5,9)$ & $5,9(3,7)$ & $241(18,0)$ & $12,5(47,8)$ \\
\hline Lanço & 9 & 510 & $43(11,2)$ & $5,0(5,5)$ & $5,5(2,7)$ & $231(18,0)$ & $36,1(33,0)$ \\
\hline Linha & 3 & 360 & $45(8,5)$ & $5,3(7,4)$ & $5,7(4,6)$ & $80(48,0)$ & $19,9(15,6)$ \\
\hline Linha & 3 & 470 & $41(7,7)$ & $5,3(8,3)$ & $6,1(3,5)$ & $159(32,5)$ & $5,0(20,6)$ \\
\hline Linha & 5 & 310 & $25(11,6)$ & $5,1(5,0)$ & $6,1(2,6)$ & $188(42,6)$ & $6,4(41,1)$ \\
\hline Linha & 7 & 530 & $36(8,5)$ & $5,2(7,9)$ & $6,1(5,1)$ & $94(36,1)$ & $14,2(42,7)$ \\
\hline Linha & 10 & 670 & $48(7,4)$ & $4,4(6,6)$ & $5,1(4,5)$ & $134(26,9)$ & $20,9(33,0)$ \\
\hline Linha & 12 & 650 & $56(10,7)$ & $4,7(9,6)$ & $5,3(6,4)$ & $212(17,4)$ & $38,0(32,6)$ \\
\hline Média & & & $(9,8)$ & $(7,0)$ & $(4,1)$ & $(29,9)$ & $(33,3)$ \\
\hline
\end{tabular}

(1) Média de 36 amostras simples; ( C.V. em \%. 
aos índices de matéria orgânica, pH em água eSMP, pois a utilização de $\boldsymbol{\alpha}=0,05$ e e $=10 \%$, na Equação (1) resulta em um número desubamostras substancialmente menor (Quadro 2).

A definição do número de subamostras, visando atender às recomendações de adubação e calagem, deve considerar a variabilidade de todos os índices de fertilidade do solo, incluídos no programa. O quadro 3 apresenta o número de subamostras, calculado pela E quação (1), para os índices de maior variabilidade, no caso o fósforo e potássio disponíveis. O número médio de subamostras cal culado com base na variabilidade do fósforo foi superior ao do potássio e reflete a maior variabilidade do primeiro (Quadro 1), principalmente quando se deseja maior confiabilidade dos resultados $(\alpha=0,05$ e e $=10 \%)$. A utilização desse critério estatístico implica, neste caso, a col eta de um número elevado de subamostras (na média 51, podendo chegar a 94).

Ressalta-se que a forma de amostragem no presente trabalho, diferenciada entre os modos de adubação para contemplar a variabilidade dos sítios de coleta, determinou um número de subamostras substancialmente maior do que o número recomendado pela Comissão... (1995) para o sistema plantio direto: 20 subamostras, em lavouras com adubação a lanço, coletadas de forma al eatória com trado, calador ou pá de corte (parte central), originando subamostras pequenas (pequeno volume), e em torno de 8 subamostras, em lavouras com adubação em linhas na largura da entrelinha, col etadas com pá de corte, originando subamostras grandes (Comissão..., 1995).

A retirada de subamostras pequenas pode apresentar problemas. Schlindwein et al. (1998) encontraram valores para fósforo disponível 30\% menores em amostragem efetuada com trado de rosca em relação à efetuada com pá de corte em lavoura com adubação em linha. Essa diferença foi atribuída à perda desolo da camada superficial, mais rica nesse nutriente. Além disto, encontraram coeficiente de variação maior (67\%) em relação à amostragem efetuada com pá de corte (33\%), refletindo a necessidade de col eta de 183 subamostras para $\boldsymbol{\alpha}=0,05$ e $\mathbf{e}=10$ e, 46 subamostras para $\alpha=0,05$ e $\mathbf{e}=20 \%$. Tais valores foram bastante superiores ao número de 44 e 11 subamostras, obtidos por esses autores pela amostragem com pá de corte, para as mesmas variações do erro e.

Cabe ainda salientar que, além dos erros de amostragem, podem ocorrer problemas em laboratório, iner entes às instal ações, equi pamentos, pessoal elimitações dos próprios métodos deanálise (Olsen \& Somers, 1982). Por exemplo, Wiethölter (1997) encontrou grande variabilidade nos resultados das análises das amostras "padrão" para fósforo disponível (Mehlich-1), entre os laboratórios da RedeOficial dos Laboratórios de Análise deSolos dos estados do Rio Grande do Sul e Santa Catarina
Quadro 2. Amplitude e média do número(1) de subamostras, considerando a variabilidade dos índices de matéria orgânica, pH e SMP de oito lavouras da região noroeste do RS, no sistema plantio direto para $\alpha=0,05$ e e $=10 \%$

\begin{tabular}{lll}
\hline \multirow{2}{*}{ Atributo } & \multicolumn{2}{c}{ Número de subamostra } \\
\cline { 2 - 3 } & Amplitude & Média \\
\hline Matéria orgânica & $2-7$ & 4,1 \\
pH em água & $1-4$ & 2,1 \\
Índice SMP & $1-2$ & 1,1
\end{tabular}

(1) $\mathbf{n}=\left[\left(\mathbf{t}_{\alpha} \cdot \mathbf{C V}\right) / \mathbf{e}\right]^{\mathbf{2}}$, sendo $\mathbf{n}$ o número de subamostras, $\mathbf{C V}$ o coeficiente de variação, t o valor da tabela do teste t para o nível de probabilidade $\boldsymbol{\alpha}$ e e é o erro em torno da média.

Quadro 3. Amplitude e média do número de subamostras calculadas(1) com base na variabilidade dos índices de potássio e fósforo disponíveis em oito lavouras da região noroeste do RS, no sistema plantio direto e probabilidades de erro

\begin{tabular}{|c|c|c|c|c|c|}
\hline \multicolumn{2}{|c|}{ Probabilidade } & \multicolumn{2}{|c|}{ Potássio (Mehlich-1) } & \multicolumn{2}{|c|}{ Fósforo (Mehlich-1) } \\
\hline $\mathbf{e}$ & $\alpha$ & Amplitude & Média & Amplitude & Média \\
\hline \multicolumn{2}{|l|}{$\%$} & \multicolumn{4}{|c|}{ _ N úmero de subamostras } \\
\hline \multirow[t]{3}{*}{10} & 0,05 & $13-95$ & 42 & $10-94$ & 51 \\
\hline & 0,10 & $9-66$ & 29 & $7-65$ & 35 \\
\hline & 0,20 & $5-39$ & 18 & $4-31$ & 21 \\
\hline \multirow[t]{3}{*}{20} & 0,05 & 3-24 & 11 & $3-24$ & 13 \\
\hline & 0,10 & $2-16$ & 8 & $2-16$ & 9 \\
\hline & 0,20 & $2-10$ & 5 & $1-6$ & 5 \\
\hline
\end{tabular}

${ }^{(1)} \mathbf{n}=\left[\left(\mathbf{t}_{\alpha} \cdot \mathbf{C V}\right) / \mathbf{e}\right]^{2}$.

(ROLAS), que apresentaram, entre 1991 e 1996, um coeficiente de variação de 44,2\%. Deste modo, como há possibilidade de ocorrer outros erros, além dos inerentes à amostragem, nas diferentes etapas do processo de recomendações de adubação e calagem pel os laboratórios, muitas vezes degranderepercussão na definição das doses desses insumos por aplicar, os limites de precisão $(\alpha=0,05$ e $\mathbf{e}=10 \%)$ utilizados nas pesquisas (Petersen \& Calvin, 1986; Santos \& Vasconcellos, 1987; Souza, 1992; Davis et al., 1995; Couto, 1997), para determinar o número de subamostras, podem ser demasiadamente exigentes para as condições de lavoura. A opção de utilizar $\alpha=0,05$ e erro $\mathbf{e}=20 \%$ apresentou um número médio de 13 e 11 subamostras para fósforo e potássio disponíveis, respectivamente, com variação, para ambos, de 3 a 24 subamostras (Quadro 3). 
O quadro 4 foi então el aborado com o objetivo de verificar o enquadramento dos valores de fósforo disponível em amostras compostas pelo número de subamostras cal culado pela Equação (1), considerando $\alpha=0,05$ e um erro $\mathbf{e}=20 \%$ em relação à média, nas classes de fósforo em função do teor deargila adotado pela Comissão... (1995). Para isso, foram retirados al eatoriamente 13 valores (Quadro 3) defósforo disponível do conjunto de 36 amostras simples de cada lavoura e calculadas as respectivas médias. Esses valores foram então comparados com a média dos valores das 36 amostras simples de cada lavoura, para verificar o enquadramento de ambos nas faixas de interpretação desse nutriente para fins de recomendação deadubação adotadas pela Comissão... (1995). A utilização de 13 subamostras para o cálculo da média não al terou as faixas de interpretação dos resultados em relação ao enquadramento das médias resultantes das 36 amostras simples.

O número de 20 subamostras éuma recomendação de rotina para o sistema convencional, sendo amplamente divulgado e exeqüível em lavoura. Esse número, que representa a fertilidade do solo dentro do critério de $\boldsymbol{\alpha}=0,05$ e $\mathbf{e}=20 \%$, é aceitável para o sistema plantio direto, desde que as subamostras contemplem a variabilidade do sítio de col eta, como utilizado no presente estudo (5 e $10 \mathrm{~cm}$ espessura e largura nas lavouras de adubação a lanço e de $5 \mathrm{~cm}$ de espessura pela largura da entrelinha de cultivo quando as adubações são nas linhas de cultivo).

\section{CONCLUSÕES}

Desde que representatividade do sítio de col eta seja atendida, como adotado neste trabalho, utilizando pá de corte na col eta das amostras com 5 e $10 \mathrm{~cm}$ de espessura elargura, respectivamente, na adubação a lanço, e $5 \mathrm{~cm}$ de espessura pela largura da entrel inha, na adubação em linhas, conclui-seque, no sistema plantio direto:

1. a variabilidade horizontal dos atributos de fertilidade do solo não se relaciona com os teores no solo e tempo de cultivo;

2. a variabilidadeé baixa para matéria orgânica, pH em água e índice SMP e alta para potássio e fósforo disponíveis;

3. pequeno número de subamostras (menor do que 8) é suficiente para representar o solo, dentro dos limites de precisão preconizados (probabilidade de erro $\alpha$ de 0,05 e erro em relação à média de $10 \%$ ) para a matéria orgânica, pH em água eíndice SMP; no entanto, o número de subamostras é el evado para potássio (média 42) e fósforo (média 51);

4. o número de subamostras (20), preconizado para a col eta de amostras no sistema convencional, admite um erro de $20 \%$ em relação à média no sistema plantio direto, não altera as faixas de interpretação dos resultados e as respectivas recomendações de adubação nesse sistema.

Quadro 4. Teores médios de fósforo (Mehlich-1) em lavouras no sistema plantio direto com diferentes características, calculados com base no número de amostras simples e faixas de interpretação adotadas pela Comissão... (1995)

\begin{tabular}{|c|c|c|c|c|c|c|}
\hline \multicolumn{3}{|c|}{ Característica da lavoura } & \multicolumn{2}{|r|}{$n=36$} & \multicolumn{2}{|c|}{$n=13^{(1)}$} \\
\hline $\begin{array}{l}\text { Modo de } \\
\text { adubação }\end{array}$ & $\begin{array}{l}\text { Tempo de } \\
\text { cultivo }\end{array}$ & $\begin{array}{l}\text { Classe de } \\
\text { solo(2) }\end{array}$ & $\begin{array}{l}\text { Valor } \\
\text { médio }\end{array}$ & $\begin{array}{c}\text { Faixa de } \\
\text { interpretação(3) }\end{array}$ & $\begin{array}{l}\text { Valor } \\
\text { médio }\end{array}$ & $\begin{array}{c}\text { Faixa de } \\
\text { interpretação(3) }\end{array}$ \\
\hline & anos & & $\mathrm{mg} \mathrm{kg}^{-1}$ & & $\mathrm{mg} \mathrm{kg}^{-1}$ & \\
\hline Lanço & 6 & 2 & 12,5 & Suficiente & 10,7 & Suficiente \\
\hline Lanço & 9 & 2 & 36,1 & Suficiente & 43,6 & Suficiente \\
\hline Linha & 3 & 3 & 19,9 & Suficiente & 18,7 & Suficiente \\
\hline Linha & 3 & 2 & 5,0 & Baixo & 4,6 & Baixo \\
\hline Linha & 5 & 3 & 6,4 & Baixo & 5,6 & Baixo \\
\hline Linha & 7 & 2 & 14,2 & Suficiente & 17,9 & Suficiente \\
\hline Linha & 10 & 1 & 20,9 & Suficiente & 20,9 & Suficiente \\
\hline Linha & 12 & 1 & 38,0 & Suficiente & 36,5 & Suficiente \\
\hline
\end{tabular}

(1) Calculado pela equação $\mathbf{n}=\left[\left(\mathbf{t}_{\alpha} \cdot \mathbf{C V}\right) / \mathbf{e}\right]^{\mathbf{2}}$, sendo $\mathbf{n}$ o número de subamostras retiradas aleatoriamente das 36 amostras simples, CV o coeficiente de variação das 36 amostras simples, $\mathbf{t}$ o valor da tabela do teste t para $\alpha=0,05$ e e $=20 \%$ em relação à média. (2) Classe 1, solo com mais de 55\% de argila; Classe 2, solos entre 41 a $55 \%$ de argila; Classe 3 , solos entre 26 a $40 \%$ de argila. (3) Interpretação dos resultados para as classes de solo, considerando o teor de argila. Classe 1, faixa suficiente: $>6,0$ mg kg-1 de P no solo; Classe 2, faixa baixo: 3,1-6,0 $\mathrm{mg} \mathrm{kg}^{-1}$ deP no solo, faixa suficiente: $>9,0 \mathrm{mg} \mathrm{kg}^{-1}$ de P no sol o; Classe 3, faixa baixo: 4,1-9,0 mg kg ${ }^{1}$ de $P$ no solo, faixa suficiente: $>14,0 \mathrm{mg} \mathrm{kg}^{-1}$ de $P$ no solo. 


\section{LITERATURA CITADA}

ANGHINONI, I. \& SALET, R.L. Amostragem do solo e as recomendações de adubação e calagem no sistema plantio direto. In: NUERNBERG, N.J ., ed. Conceitos efundamentos do sistema plantio direto. Lages, Sociedade Brasileira de Ciência do Solo/Núcleo Regional Sul, 1998. p.27-52.

ANGHINONI, I. \& VOLKWEISS, S.J. Recomendações de uso de fertilizantes no Brasil. In: ESPINOSA, W. \& OLIVEIRA, A.J. SIMPÓSIO NA AGRICULTURA BRASILEIRA, Brasília, 1984. Anais. Brasília, Empresa Brasileira de Pesquisa Agropecuária, 1984. p.179-204.

BAYER C. Dinâmica da matéria orgânica em sistemas de manejo de solo. Porto Alegre, Universidade Federal do Rio Grande do Sul, 1996. 240p. (Tese de Doutorado)

COMISSÃO DE FERTILIDADE DO SOLO - CFSRS/SC. Recomendações de adubação e calagem para os estados do Rio Grande do Sul e Santa Catarina. 3.ed. Passo Fundo, SBCS - Núcleo Regional Sul/E MBRAPA/CNPT, 1995. 224p.

COUTO, E.G. Variabilidade espacial de propriedades do solo influenciadas pela agricultura em escala regional e local no sul do estado do Mato Grosso. Porto Alegre, Universidade Federal do Rio Grande do Sul, 1997. 183p. (Tese de Doutorado)

DAVIS, J.G.; HOSSNER, L.; WILDING, L.P. \& MANU, A. Variability of soil chemical properties in two sandy Dunal soil of Niger. Soil Sci., 159:321-330, 1995.

DENARDIN, J.E.; KOCHHANN, R.A.; PÖTTKER, D.; WIETHÖLTER, S.; FAGANELLO, A.; SATTLER, A.; PORTELLA, J .A.; VOSS, M.; BEN, J .R.; BERTON, A.L.; MELO, I.B. \& CIPRANDI, M.A.O. Projeto Metas - Um caso de sucesso na aplicação do modelo de pesquisa e desenvolvimento. In: REUNIÃO SUL BRASILEIRA DE CIÊNCIA DO SOLO - MANEJ O SUSTENTÁVEL DO SOLO, 2., Santa Maria. 1998. Resumos Expandidos. Santa Maria, Sociedade Brasileira de Ciência do Solo/Núcleo Regional Sul, 1998. p.263-268.

EMPRESA BRASILEIRA DE PESQUISA AGROPECUÁRIA EMBRAPA. Centro Nacional de Pesquisa do Solo. Sistema Brasileiro de Classificação de Solos. Rio de J aneiro, 1999. $412 \mathrm{p}$.

FARIAS, A.D. \& FERREIRA, T.N. Expansão do sistema plantio direto no Rio Grande doSul. Inf. EMATER - RS, 8:1-3, 1997.

J AMES, D.W. \& DOW, A.I. Source and degree of soil variation in the field: the problem of sampling for soil test and estimating soil fertility status. Washington, 1972. 749p. (Agricultural Experiment Station Bulletin)

KLEPKER, D. \& ANGHINONI, I. Phosphate uptake and corn root distribuition as affected by fertilizer placement and soil tillage. Agronomy-Trends in Agril. Sci., 1:111-115, 1993.

KLEPKER, D. \& ANGHINONI, I. Características físicas e químicas do solo, afetadas por métodos de preparo e modos de adubação. R. Bras. Ci. Solo, 19:395-401, 1995.
KRAY, C.H.; SALET, R.L. \& ANGHINONI, I. Variabilidade horizontal eamostragem dirigida do sol o no sistema plantio direto. Porto Alegre, Universidade Federal do Rio Grande do Sul, 1997. 6p. (Relatório de Pesquisa)

OLSEN, S.R. \& SOMMERS, L.E. Phosphorus. In: PAGE, A.L.; MILLER, R.H.\& KEENEY, D.R. Chemical and microbiological properties. 2. ed. Madison, Soil Science Society of America, 1982. p.403-427.

PETERSEN, R.G. \& CALVIN, L.D. Sampling. In: KLUTE, A., ed. Methods of soil analysis: part 1. Physical and mineralogical methods. 2.ed. Madison, American Society of Agronomy, 1986. p.33-51.

SALET, L.R.; KRAY, C.H.; FORNARI, T.G.; CONTE,E.; KOCHHANN, R.A. \& ANGHINONI, I. Variabilidade horizontal e amostragem de solo no sistema de plantio direto. In: REUNIÃO SUL BRASILEIRA DE CIÊNCIA DO SOLO, 1., Lages, 1996. Resumos Expandidos. Lages, Sociedade Brasileira de Ciência do Solo/Núcleo Regional Sul, 1996. p.74-76.

SANTOS, H.L. \& VASCONCELLOS, C.A. Determinação do número de amostras de solo para análise química em diferentes condições de manejo. R. Bras. Ci. Solo, 11:97100, 1987.

SCHLINDWEIN, J.A.; SALET, L.R. \& ANGHINONI, I. Variabilidade dos índices de fertilidade do solo no sistema plantio direto e col eta de amostras representativas de solo. In: REUNIÃO BRASILEIRA DE FERTILIDADE DO SOLO E NUTRIÇÃO DE PLANTAS, 23.; REUNIÃO BRASILEIRA SOBRE MICORRIZAS, 7.; SIMPÓSIO BRASILEIRO SOBRE MICROBIOLOGIA DO SOLO, 5.; REUNIÃO BRASILEIRA DE BIOLOGIA DO SOLO, Caxambú, 1998. Resumos. Caxambú, Sociedade Brasileira de Ciência do Solo, 1998. p.265.

SOLLINS, P.; HOMANN, P. \& CALDWELL, B.A. Stabilization and destabilization of soil organic matter: mechanisms and controls. Elsevier Sci., 74:65-105, 1996.

SOUSA, D.G.M. \& VOLKWEISS, S.J. Efeito residual do superfosfato triplo aplicado em pó e em grânulos. R. Bras. Ci. Solo, 11:141-146, 1987.

SOUZA, L.S. Variabilidade espacial do solo em sistemas de manejo. Porto Alegre, Universidade F ederal do Rio Grande do Sul, 1992. 102p. (Tese de Doutorado)

TEDESCO, M.J .; GIANELLO, C.; BISSANI, C.A.; BOHNEN, H. $\&$ VOLKWEISS, S.J. Análise de solo, plantas e outros materiais. 2.ed. Porto Alegre, Universidade Federal do Rio Grande do Sul, 1995. 147p. (Boletim Técnico, 5)

WIETHÖLTER, S. Controle de qualidade de análises de solo da ROLAS. In: CONGRESSO BRASILEIRO DE CIÊNCIA DO SOLO, 26., Rio de J aneiro, 1997. Anais. Rio de J aneiro, Sociedade Brasileira de Ciência do Solo, 1997. CD-ROM.

WIETHÖLTER, S.; BEN, J .R.; KOCHHANN, R. \& PÖTKER, D. Fósforo e potássio no solo no sistema plantio direto. In: NUERNBERG, N.J ., ed. Conceitos e fundamentos do sistema plantio direto. Lages, Sociedade Brasileira de Ciência do Solo/Núcleo Regional Sul, 1998. p.121-149. 